\title{
La différence des pertes de charge dans un écoulement en conduit circulaire, d'une part, et dans un écoulement en canal à ciel ouvert, à faible tirant d'eau, d'autre part
} The difference between the head losses in a pipe with a circular cross section and those in a shallow open channel

\author{
PAR M. CHEN CHE-PEN \\ ATTACHÉ DE RECHERCHE AU C.N.R.S. \\ Communication présentée à la Société Hydrotechnique de France, \\ au cours de sa séance du 19 juin 1959
}

Les mesures de répartition de vitesse près de la paroi d'un canal rectangulaire lisse montrent qu'il n'y existe aucune différence et que l'éco'ulement dans cette zone suit la mème loi universelle $\left.u / u_{*}=g(y) u_{*} / v\right)$ que dans les conduits circulaires. Mais la loi de répartition de vitesse loin de la paroi est certainement bealcoup plus complexe et probablement de la forme :

$\left(u-\mathrm{U}_{m}\right) / u_{*}=\mathrm{G}\left(\mathscr{H}, \chi / b, \lambda, y / \mathrm{D}_{\mathrm{HI}}, z / D_{\mathrm{HI}}\right)$

Par conséquent, on pent en déduire que le coefficient de perte de charge pour les canaux est de la méme forme que $\lambda^{\prime}$ correspondant aux conduits circulaires, at une constante prés, c'est-ì-dire :

$$
1 / \sqrt{\lambda}-1 / \sqrt{\lambda^{\prime}}=\mathrm{G}_{1}(\mathscr{y}, \chi / b, \lambda)
$$

Dans le cas d'un canal rectangulaire infiniment large, à faible nombre de Fronde, on pent obtenil une forme très simple de $\mathrm{G}_{1}$ :

$$
1 / \sqrt{\lambda}-1 / \sqrt{\lambda^{\prime}}=a \mathscr{H}-b
$$

Velocity distribution measurements near the walls of a smooth rectangular channel show that there is no difference and that flow in this region is subject to the same general law as applies in pipes with circular cross sections, i.e., $n / u_{*}=$ o $\left(y l_{*} / v\right)$. However, the velocity distribution at some distance from the walls is certainly more complicated and probably takes the following form:

$\left(u-\mathrm{U}_{m}\right) / u_{*}=\mathrm{G}\left(\mathscr{H H}, \chi / b, \lambda, y / \mathrm{D}_{\mathrm{H}}, z / D_{\mathrm{II}}\right)$ It can therefore be dednced that the head loss coefficient for channels has the same form as $\lambda^{\prime}$ for circular pipes except for a constant, i.e.

$$
1 / \sqrt{\lambda}-1 / \sqrt{\lambda^{\prime}}=\mathrm{G}_{1}(\mathscr{\mathscr { X }}, \chi / b, \lambda)
$$

An infinitely wide channel with a low Froude number gives a very simple expression for $\mathrm{G}_{1}$, i.e,

$$
1 / \sqrt{\lambda}-1 / \sqrt{\lambda^{\prime}}=a \mathfrak{H}-b
$$

\section{NOTATIONS}

$\rho:$ Masse volumique du fiuide;

$\nu$ : Viscosité cinématique;

$r_{0}$ : Rayon du conduit;

$h$ : Profondeur d'eau;

$\%$ : Périmètre mouillé;

$b$ : Largeur à la surface libre;

$\mathrm{D}_{\mathrm{H}}$ : Diamètre hydraulique $=4 \mathrm{R}_{\mathrm{H}}=4 \mathrm{~S} / \%$;

$y$ : Distance à la paroi;

$u$ : Vitesse du fluide en un point $y$;

$\mathrm{U}$ : Vitesse moyenne dans la section $=$ Débit/Section;
$\mathrm{U}_{m}$ : Vitesse maximum;

$\tau_{0}$ : Force de frottement sur la paroi;

$u_{*}:$ Vitesse de frottement $=\sqrt{\tau_{0} / \wp}$;

$c_{j}$ : Coefficient de frottement;

$\lambda$ : Coefficient de perte de charge dans les canaux;

$\lambda^{\prime}$ : Coefficient de perte de charge dans les conduits circulaires;

$g$ : Accélération de la pesanteur;

$\mathcal{R}:$ Nombre de Reynolds $=\mathrm{D}_{\mathrm{rI}} \mathrm{U} / \mathrm{v}$;

$\mathscr{H}:$ Nombre de Froude $=\mathrm{U} / \sqrt{g h}$;

$k$ : Paramètre de rugosité. 
Depuis les expériences de Niliuradse et les travaux de Von Karman, le problème de l'écoulement dans les conduits cylindriques peut être considéré comme résolu. Mais celui de l'écoulement dans les canaux découverts reste toujours dans l'état stationnaire avec ses formules empiriques, c'est-à-dire malgré de nombreuses recherches thériques et experimentales depuis trente ans; pratiquement on n'a pas fait de progrès sensible. Néanmoins, le mécanisme de ces deux types d'écoulement ne doil pas être très différent. On a essayé de transposer la loi de l'écoulement dans les conduits circulaires à l'écoulement dans les canaux découverts; mais le coefficient de perte de charge $\lambda$ ainsi obtenu en prenant le diametre hydraulique $D_{I I}$ à la place de $D$ représente seulement le cas limite le plus favorable [1].

La raison de ces échees réside probablement dans le fait que l'on s'atlache trop à la rugosité pour ces recherches, ceci à cause de son importance pour les applications industrielles. Or, dans les canaux d'essai, la réalisation de la rugosité du type Nikuradse est techniquement plus difficile, le régime de l'écoulement (lisse, rugueux ou transitoire) moins certain, et les mesures peuvent rarement ètre effectuées avec la mème précision. Ainsi, nous bornons-nous à traiter ce problème d'abord dans le cas de la paroi lisse, théoriquement et expérimentalement. Puis nous verrons comment le résultat ainsi obtenu pourra s'étendre aux canaux rugueux.

Avant d'aborder les canaux, nous ferons un rappel rapide sur les lois de la répartition de vilesse et de la perte de charge pour les conduits cylindriques de section circulaire [2].

1" Près de la paroi, l'écoulement ne dépend que des conditions à la paroi. Le profil de ritesse $u(y)$ est défini par la force de frottement $\tau_{0}$ ou par la vitesse de frottement :

$$
u_{*}=\sqrt{\tau_{0} / \rho}
$$

On a alors:

$$
\frac{u}{u_{*}}=g\left(\frac{y / u_{*}}{*}\right)
$$

En fait, cette loi est universelle, mais a plusieurs expressions selon les valeurs de $y u_{*} / \nu$ :

$$
\text { a) } \frac{y u_{*}}{y}<5 \quad \frac{u}{u_{*}}=\frac{y u_{*}}{y}
$$

$$
\begin{aligned}
& \text { b) } 5<\frac{y u_{*}}{\nu}<25 \quad \frac{u}{u_{*}}=g\left(\frac{y u_{*}}{\nu}\right) \\
& \text { c) } \frac{y u_{*}}{\nu}>25 \quad \frac{u}{u_{*}}=5,65 \log _{10} \frac{y u_{*}}{\nu}+4,85
\end{aligned}
$$

$2^{\circ}$ Loin de la paroi, l'écoulement est indépendant des particularilés de l'écoulement près de la paroi et de la répartition des forces de frottement, mais défini par la force de frottement totale (ou moyenne) dans la section.

La viscosité du fluide n'intervient pas.

$$
\frac{u-\mathrm{U}_{m_{*}}}{u_{*}}=f\left(\frac{y}{r_{0}}\right)
$$

A la limite de ces deux zones il existe une zone commune où les deux lois (4) et (5) sont toutes les deux valables, et ou la fonction $f$ est nécessairement de la forme logarithmique, c'està-dire :

$\operatorname{pour}\left(y / r_{0}\right) \leq 0,2$ :

$$
\frac{u-\mathrm{U}_{m}}{u_{*}}=5,65 \log _{10} \frac{y}{r_{0}}-1,24
$$

De (4) et (6), on arrive à tirer une formule de perte de charge sous la forme:

$$
\frac{1}{\sqrt{\lambda^{\prime}}}=2 \log _{10} \propto \sqrt{\lambda^{\prime}}-0,8
$$

en passant par le caleul de la vitesse moyenne $U$ et par la définition de :

$$
\tau_{0}=c_{f} \cdot \frac{\mathrm{U}^{2}}{2}=-\frac{\lambda^{\prime}}{4} \cdot \frac{\mathrm{U}^{2}}{2}
$$

Revenons maintenant au cas des canaux découverts. L'idée directrice de la présente étude se base justement sur l'existence des deux zones distinctes ci-dessus mentionnées.

1" Près de la paroi, les lois de répartitions de vitesses sont exactement les mêmes d'après nos essais :

$$
\frac{u}{u_{*}}=g\left(\frac{y u_{*}}{\nu}\right)
$$

2" Loin de la paroi la viscosité n'intervient pas non plus. Mais la loi de répartition de vitesse est plus complexe en raison de la na- 


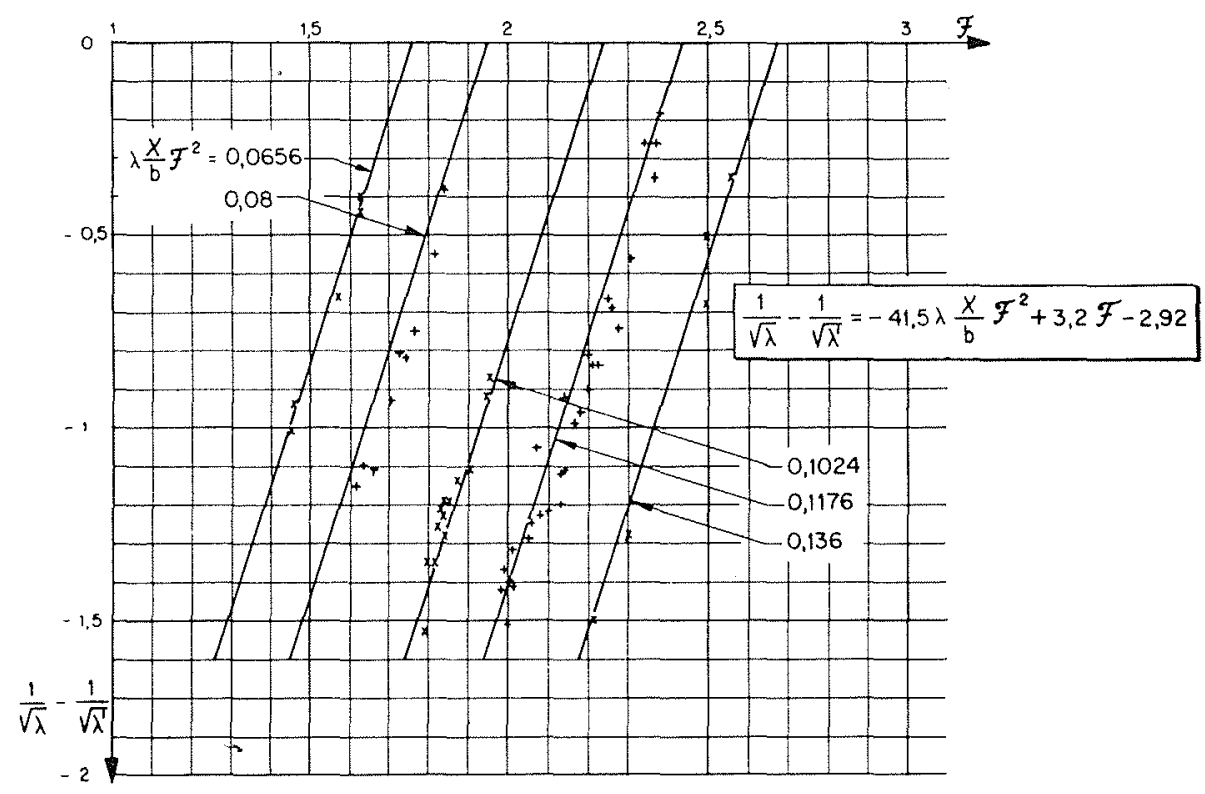

Fig. 1

ture de l'écoulement et de la forme de sa section:

$$
\frac{u-\mathrm{U}_{m}}{u_{*}}=\mathrm{G}\left(\frac{y}{\mathrm{D}_{\mathrm{HI}}}, \frac{z}{\mathrm{D}_{\mathrm{IX}}}, \lambda, \frac{\chi}{b}, \mathscr{H}\right)
$$

Cette dernière pourra se simplifier dans le cas d'un canal infiniment large rectangulaire, ce qui permet d'établir une formule de $\lambda$ à partir de la répartition de vitesse.

1" Près de la paroi, pour $\left(y u_{*} / y\right)>25:$

$$
\frac{u}{u_{*}}=5,65 \log _{10} \frac{y J u_{*}}{v}+4,85
$$

$2^{\circ}$ Loin de la paroi :

$$
\frac{u-\mathrm{U}_{m}}{u_{*}}=\mathrm{C}\left(\frac{y}{h}, \mathscr{T}\right)
$$

Dans la zone commune, (10) s'écrit sous la forme suivante :

pour $(y / h) \leq 0,1$ :

$$
\frac{u-\mathrm{U}_{m}}{u_{*}}=5,65 \log _{10} \frac{y}{h}+\mathrm{A}(\mathscr{F})
$$

A est une fonction du nombre de Froude.

De (4) et (11), on tire :

$$
\frac{\mathrm{U}_{m}}{u_{*}}=5,65 \log _{10} \frac{h u_{*}}{\nu}+4,85-\mathrm{A}
$$

La vitesse moyenne $U$ est. liée à $U_{m}$ par la relation :

$$
\begin{array}{r}
\frac{\mathrm{U}}{u_{*}}=\int_{0}^{1} \frac{u}{u_{*}} . d\left(\frac{y}{h}\right) \\
=\frac{\mathrm{U}_{m}}{u_{*}}+\int_{0}^{1} \mathrm{G}\left(\frac{y}{h}, \mathscr{H}\right) d\left(\frac{y}{h}\right) \\
=\frac{\mathrm{U}_{m}}{u_{*}}+\mathrm{B}(\mathfrak{W})
\end{array}
$$

ce ([ui donne :

$$
\frac{\mathrm{U}}{u_{*}}=5,65 \log _{10} \frac{h u_{*}}{\nu}+\mathrm{B}_{1}-\mathrm{A}_{1}
$$

or :

d'où :

$$
u_{*}=\sqrt{\frac{\tau_{0}}{\rho}}=\sqrt{\frac{\lambda}{8}} \mathrm{U}
$$

$$
\frac{1}{\sqrt{\lambda}}=2 \log _{10} \alpha \sqrt{\lambda}+A_{2}
$$

En vertu de (7):

$$
\frac{1}{\sqrt{\lambda}}-\frac{1}{\sqrt{\lambda^{\prime}}}=2 \log _{10} \sqrt{\frac{\lambda}{\lambda^{\prime}}}+\mathrm{A}_{3}(\mathscr{g})
$$

$\lambda$ et $\lambda^{\prime}$ étant peu différents, on peut done négliger le premier terme du second membre de (16).

Les essais ont d'abord été effectués à SaintCyr dans un canal vitré inclinable, de section rectangulaire de $35 \mathrm{~cm} \times 25 \mathrm{~cm}$ et de $12 \mathrm{~m}$ de long. Voici le résultat obtenu :

$\frac{1}{\sqrt{\lambda}}-\frac{1}{\sqrt{\lambda^{\prime}}}=-41,5 \lambda \cdot \frac{\chi}{b} \mathscr{g}^{2}+3,2 \mathscr{F}-2,92$ 


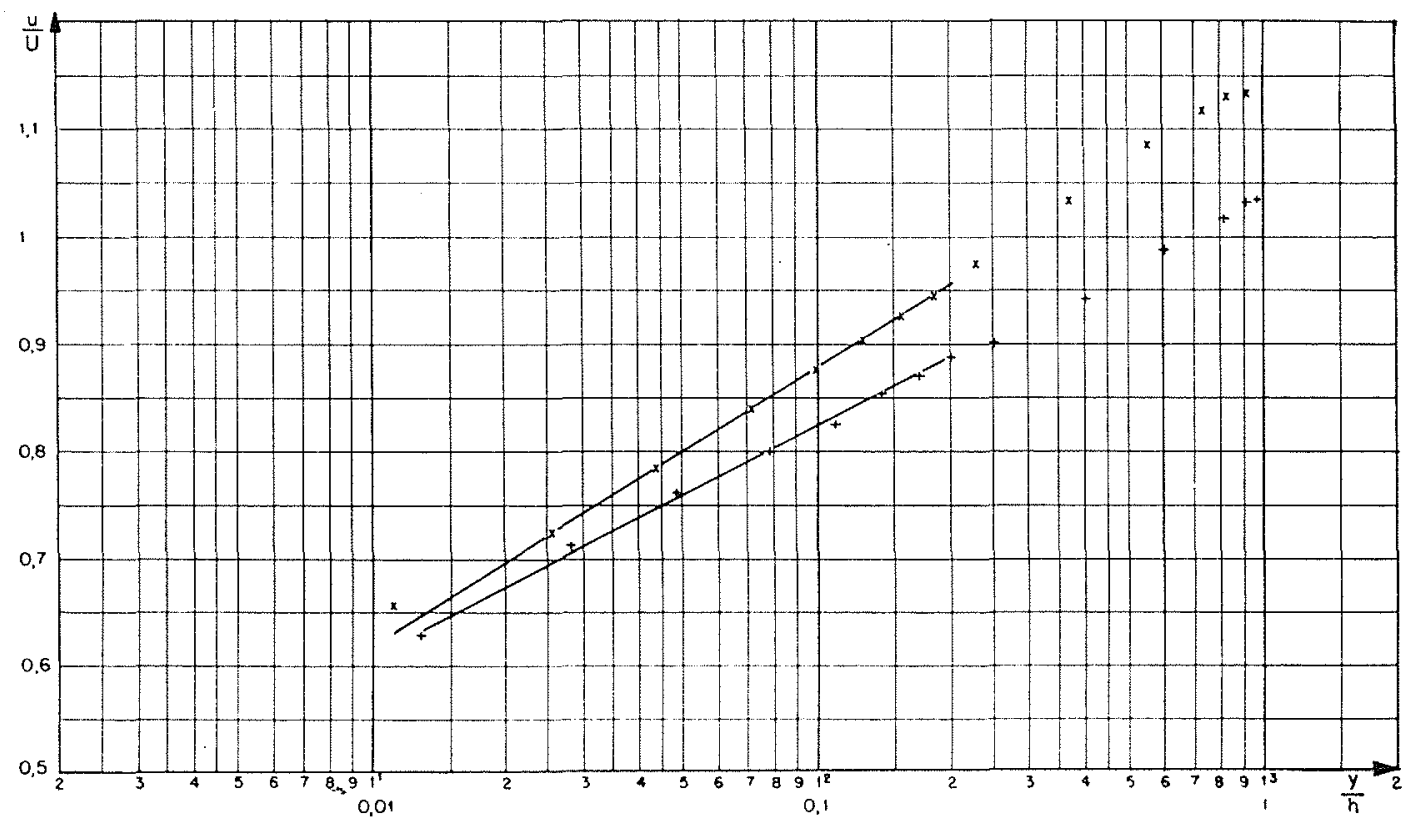

FIG. 2

Répartition de vitesse près des parois

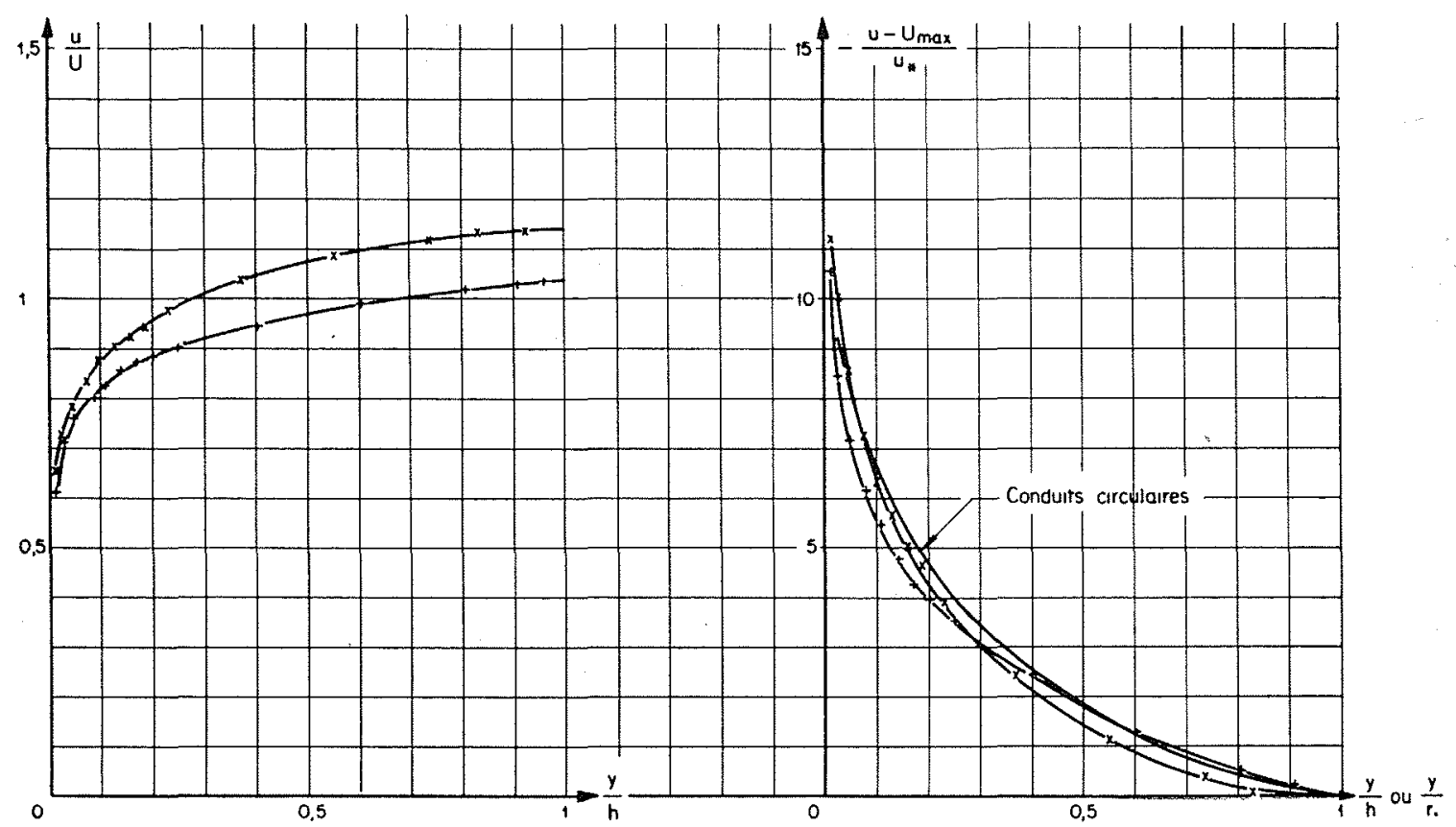

FIG. 3

Répartition de vitesse loin des parois 

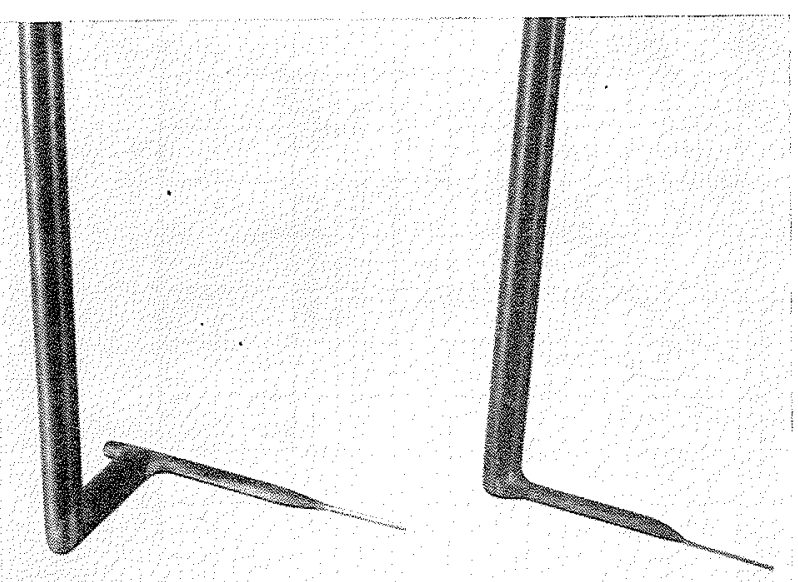

PIG. 4

On trouvera les points expérimentaux sur la figure 1.

Dans le cas de l'éconlement fluvial à grand nombre de Reynolds, dans un canal très large à faible tirant d'eau, le premier terme du second

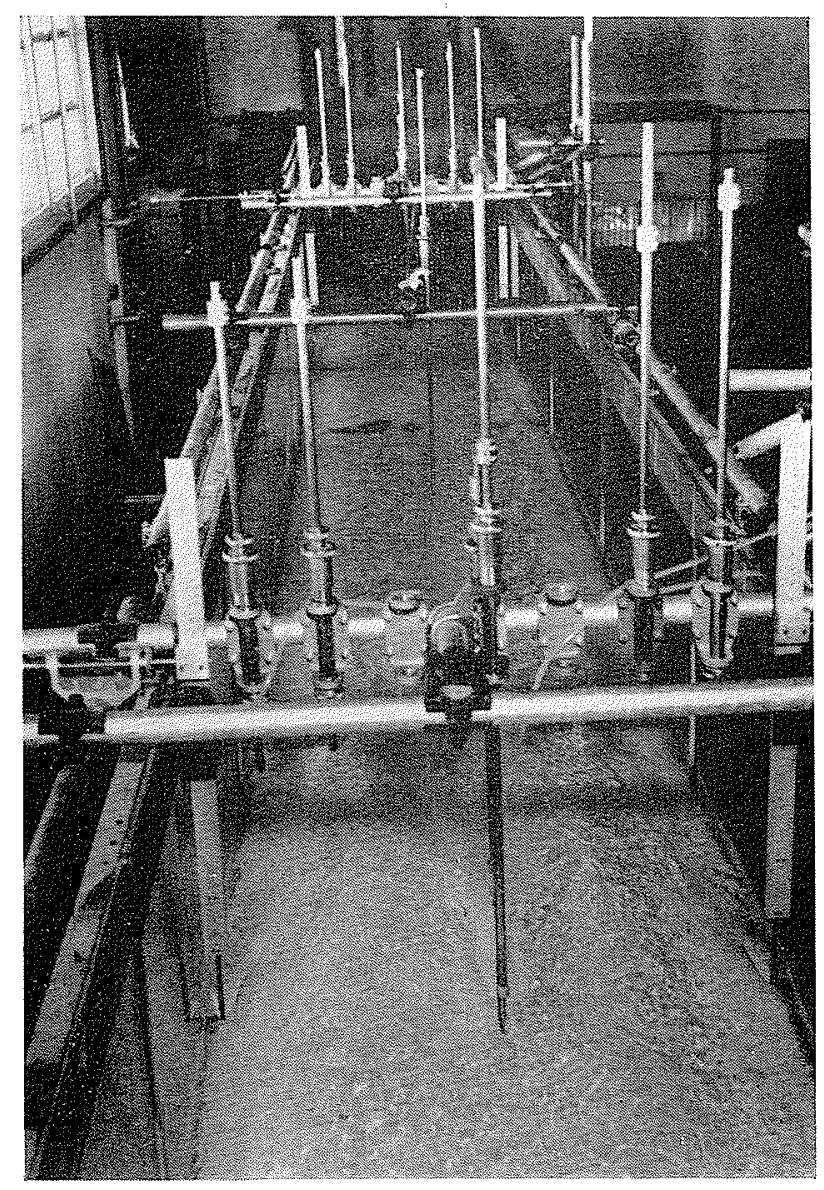

Fig. 5 membre est négligeable, et cette expression se réduit à :

$$
\frac{1}{\sqrt{\lambda}}-\frac{1}{\sqrt{\lambda}}=3,2 g-2,92
$$

Afin de vérifier la validité de (17), nous avons récemment entrepris deux séries d'essais au Laboratoire National d'Hydraulique à Chatou dans un canal à parois latérales vitrées, de section rectangulaire de $1,02 \mathrm{~m}$ de large, et de $21 \mathrm{~m}$ de long. Le fond est en ciment lissé dans la première serie d'essais, et enduit de peinture, pour être encore plus lisse, dans la seconde série. Il a une pente fixe de $1 \%$.

Les mesures de répartition de la vilesse confirment bien ce que nous avons exposé plus haut. La profondeur d'eau élant de $10 \mathrm{~cm}$ environ, on peut assimiler l'écoulement dans la partie centrale du canal au cas du canal infiniment large rectangulaire. On trouvera sur les figures 2 et 3 les courbes de répartitions de vitesse suivant la verticale passant par l'axe du canal, ainsi que celle obtenue dans les conduits circulaires [3].

Ces mesures sont faites à l'aide de tubes de

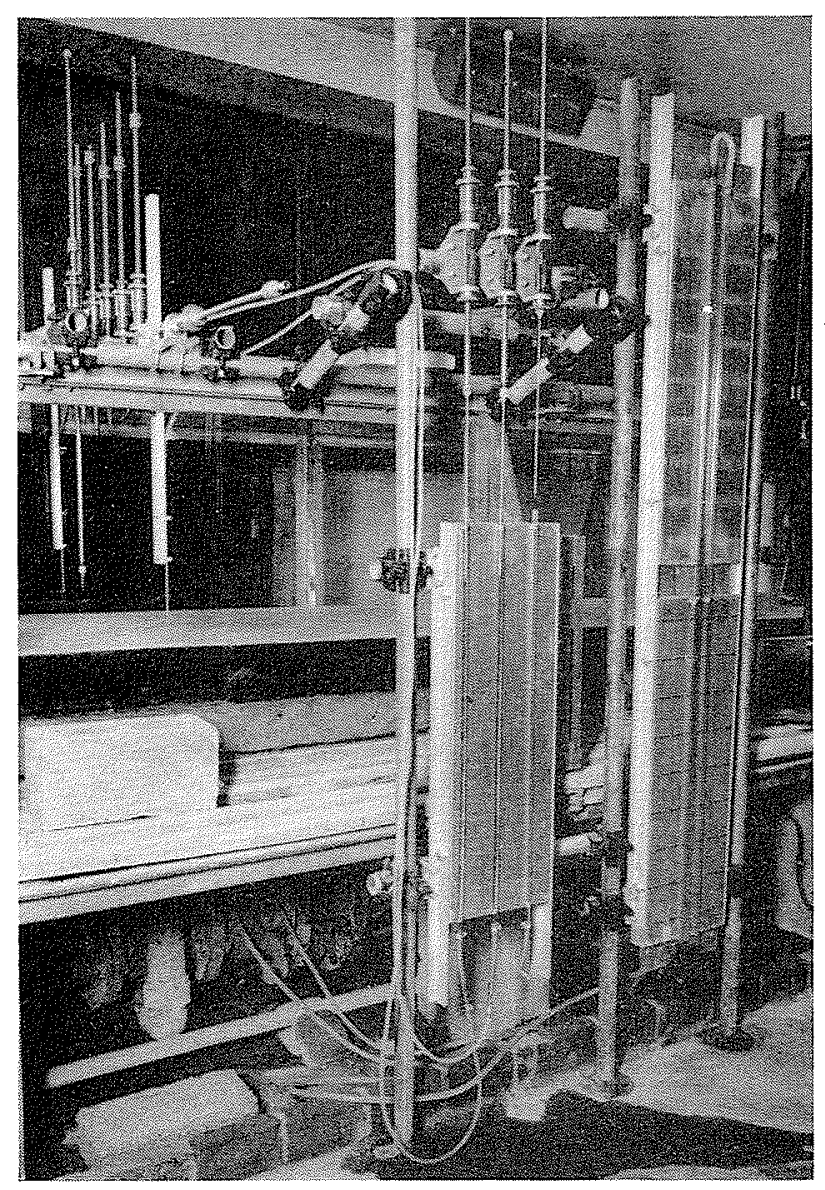

Fili. 6 
Pitot aplatis de $5 / 10^{\circ}$ de $m$ d'épaisseur avec une fente de $1 / 10^{\circ}$ de $\mathrm{mm}$ environ (fig. 4). Les vitesses indiquées sont les moyennes de 5 mesures consécutives avec un intervalle de temps déterminé. Les photos $n^{\circ 5} 5$ et 6 donneront une vue d'ensemble de l'installation.

Remarquons enfin que les nombres de Reynolds de ces essais varient de 150000 à 750000 , et que le second membre de (17) ou de (18) reste toujours négatif ou, à la limite, nul. De nombreuses expériences ont été effectuées par d'autres chercheurs [4]. Elles semblent conduire à des résultats qui ne concordent pas avec la formule (17), mais les nombres de Reynolds atteints au cours de ces expériences sont toujours inférieurs à $10^{5}$.

Bien que nos essais aient été effectués en canaux lisses, le résultat s'étend aux canaux ru- gueux. Pour donner un exemple, nous avons appliqué la formule (18) au canal de force de Kembs, de $800 \mathrm{~m}$ de long et de section trapézoïdale $(40 \mathrm{~m}$ au plafond, berges à $2 / 1$, tirant d'eau de 8 à $9 \mathrm{~m}$ ). Les pertes de charge mesurées donnent, en appliquant la formule (18), une rugosité de $0,25 \mathrm{~mm}$, ce qui correspond assez bien aux berges de béton lissé. On trouverait $k=5 \mathrm{~mm}$ environ, si l'on appliquait simplement la formule de perte de charge des conduits circulaires qui est, pour le régime complètement rugueux :

$$
\frac{1}{\sqrt{\lambda^{\prime}}}=2 \log \frac{\mathrm{D}_{\mathrm{II}}}{k}+1,14
$$

Les résultats sont résumés dans le tableau ciaprès :

\section{CANAL DE FORCE DE KEMBS}

Tronçon $\mathrm{km} 4,95-5,75$. - Longueur : $800 \mathrm{~m}$

\begin{tabular}{|c|c|c|}
\hline ' DATE DE JAUGEAGE & Juillet 1946 & Mai 1947 \\
\hline Débit $Q \ldots \ldots \ldots \ldots \ldots \ldots$ & $674 \mathrm{~m}^{3} / \mathrm{s}$ & $940 \mathrm{~m}^{3} / \mathrm{s}$ \\
\hline Profondeur d'eau $h . . \ldots \ldots$. & $8,23 \mathrm{~m}$ & $9,31 \mathrm{~m}$ \\
\hline Section mouillée $\mathrm{s} \ldots \ldots \ldots \ldots \ldots \ldots \ldots$ & $473 \mathrm{~m}^{2}$ & $827 \mathrm{~m}^{2}$ \\
\hline Périmètre mouillé $\chi \ldots . .$. & $76,2 \mathrm{~m}$ & $93,8 \mathrm{~m}$ \\
\hline Diametre hydraulique $\mathrm{D}_{\mathrm{II}}=4 \mathrm{~S} / \% \ldots \ldots \ldots \ldots$ & $24,82 \mathrm{ml}$ & $35,26 \mathrm{~m}$ \\
\hline Largeur de la surface libre $b \ldots \ldots \ldots \ldots \ldots$ & $72,2 \mathrm{~m}$ & $89 \mathrm{~m}$ \\
\hline Vitesse moyenne $u=Q / \mathrm{S} \ldots \ldots \ldots$ & $1,425 \mathrm{~m} / \mathrm{s}$ & $1,137 \mathrm{~m} / \mathrm{s}$ \\
\hline Perte de charge $\Delta H$ entre les deux sections...... & $43 \mathrm{~mm}$ & $19 \mathrm{~mm}$ \\
\hline$j=\Delta \mathrm{H} / \mathrm{L} \ldots$ & $53,75 \cdot 10^{-6}$ & $23,75 \cdot 10^{-15}$ \\
\hline$\lambda=\frac{\mathrm{D}_{\Pi}}{u^{2} / 2 g} \cdot j \ldots \ldots \ldots \ldots \ldots \ldots \ldots \ldots$ & 0,0129 & 0,0127 \\
\hline Nombre de Reynolds $\mathfrak{R}=u . \mathrm{D}_{\mathrm{H}} / \nu \ldots \ldots \ldots \ldots$ & $35,4.10^{6}$ & $40.10^{6}$ \\
\hline Nombre de Froude $\mathscr{H}=u / \sqrt{g h}$ & 0,1587 & 0,119 \\
\hline$k$ par $(18) \ldots$ & $0,229 \mathrm{~mm}$ & $0,259 \mathrm{~mm}$ \\
\hline$k$ par (19) en supposant $\lambda=\lambda^{\prime}$. & $3,67 \mathrm{~mm}$ & $4,81 \mathrm{~mm}$ \\
\hline
\end{tabular}

Cette étude a été réalisée sous la direction du Professeur A. Fortier, et les essais à Chatou ont pu être effectués grâce au concours de l'Electricité de France; qu’il me soit permis de leur exprimer ici ma profonde reconnaissance et mes remerciements, Les détails des essạis seront publiés in extenso prochainement. 


\section{RÉFÉRENCES}

[1] hirschmer (O.). - Perte de charge dans les conduites forcées et les canaux découverts, Revule Générale de l'Hydrallique, n 51, mai-juin 1949.

[2] Fonten (A.). - Cours d'Hydrodynamique Supérieure à la Sorbonne.

3] Bakmeterr (Boris A.). -- Mécanique de l'écoulement turbulent des fluides. Traduction de Jorissen (A.). - Ed. Dunod.

4] Marchi (Enrico), - Le mouvement uniforme dans les courants à surface libre. La Ricerca Scientifica, août 1956.
-. Strauj (Lorenz G.), Shberman (Edward) et Nelson (Herbert C.). - Some observations on open channel flow at small Reynolds numbers. - Journal of the Engineering Mechanics Division, Proceedings of the American Society of Civil Fng. (Proc. paper 1031.)

- Petersen (M. S.), MacGee (H. C.), Fenwick (G. B.). Triangular flume study of distortion effects. Research report Nr. 2-1, Report Nr.1. U.S. Army Engineers Waterways Experiment Station, CE, Vicksburg, Miss., September 1957.

\section{IS C US S I O N}

\section{Président : M. Fontrer}

I. le Président remercie M. Chex Che Pex de son exposé. Il souligne son très gros effort expérimental et son mérite d'aroir mis en évidence, par des mesures de vitesses et de pertes de charge extrêmement soignées, les différences entre $\lambda^{\prime}$, coefficient de pertes de charge du conduit de section circulaire, et $\lambda$, coefficient de pertes de charge dans un canal : en effet, si ces differences sont extremement faibles dans la plupart des cas pratiques pour de petits nombres de Reynolds, on ne pent en conclure qu'elles sont toujours négligeables.

M. Remenenas souligne l'intérèt pratique de l'étude de M. Chrs, qui donne pour la première for l'explication rationnelle d'un phénomène resté obscur. Diverses mesures avaient déjà montré aux ingénieurs que le coefficient de Strickler d'une galerie, comme la galerie de dérivation provisoire de Génissiat, d'une part et d'un canal a ciel ouvert à faible tirant d'eau relatif, comme le canal de Donzère-Mondragon, d'autre part, étaient très différents pour une même valeur du rayon hydraulique, de la vitesse de l'eau et de la rugosité des parois $(k=100$ dans une grande galerie contre $k \# 60$ pour un canal à ciel ouvert « homologué »). C'est en vue de préciser ce phénomène qu'ont été effectués par E.D.F. les essais sur le canal de Kembs dont à parlé M. Chen.

On peut vérifier que le frottement de l'air sur la surface libre ne peut rendre compte du supplément de pertes de charge observé; lorsque cette surface est ondulée comme cela se produit lorsque le tirant d'eau est voisin de la profondeur critique, il se peut qu'il en résulte des per'tes supplémentaires dont le terme $\mathscr{F}^{2}$ qui figure dans les formules de M. Ches pourrait éventuellement rendre compte.

M. le Président indique que ces différences tiennent à ce que les formules du type Strickler ont été établies dans un domaine limite de nombres de Reynolds et ne peuvent donc etre extrapolées à de grands nombres de reynolds. La différence entre l'écoulement dans un canal et l'écoulement dans un tuyau réside dans les courbes de répartition des vitesses qui traduisent des phénomenes identiques dans les deux cas près de la paroi, mais divergent très sensiblement dans la partie centrale de ces écoulements.

Le premier terme de l'équation donnée par M. CHeN est négligeable dans la plupart des applications, mais il ne devient plus négligeable lorsqu'on est en écoulement torrentiel. Il est peut-être lié à l'existence des ondulations de surface, dont a parlé $M$. Remenieras.

En réponse à une question de M. SAint-GuiLY, M. lo l'lésident indique que l'expression de la différence des coeffieients de perte de charge en fonction du nombre de Froude est d'origine purement expérimentale.

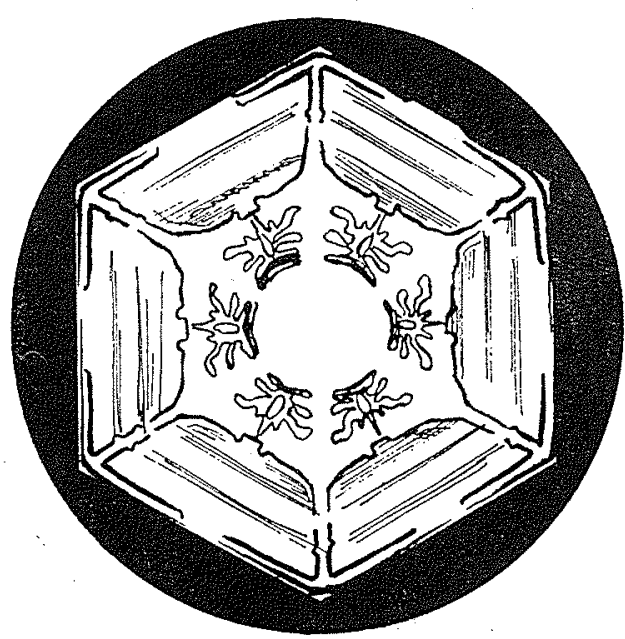

